\title{
The cardiopulmonary reflexes of spontaneously hypertensive rats are normalized after regression of left ventricular hypertrophy and hypertension
}

T.A. U ggere, G.R. Abreu, K.N. Sampaio, A.M. Cabral and N.S. Bissoli

\author{
Departamento de Ciências Fisiológicas, Centro Biomédico, \\ Universidade Federal do Espírito Santo, Vitoria, ES, Brasil
}

\section{Correspondence \\ N.S. Bissoli \\ Departamento de Ciências \\ Fisiológicas, CBM, UFES \\ Av. Marechal Campos, 1468 \\ 29040-090 Vitoria, ES \\ Brasil \\ Fax: $+55-27-335-7330$}

Presented at the XIV Annual Meeting of the Federação de Sociedades de Biologia Experimental, Caxambu, MG, Brasil, August 25-28, 1999

Received April 9, 1999

Accepted February 4, 2000

\section{Abstract}

Cardiopulmonary reflexes are activated via changes in cardiac filling pressure (volume-sensitive reflex) and chemical stimulation (chemosensitive reflex). The sensitivity of the cardiopulmonary reflexes to these stimuli is impaired in the spontaneously hypertensive rat (SHR) and other models of hypertension and is thought to be associated with cardiac hypertrophy. The present study investigated whether the sensitivity of the cardiopulmonary reflexes in SHR is restored when cardiac hypertrophy and hypertension are reduced by enalapril treatment. Untreated SHR and WKY rats were fed a normal diet. Another groups of rats were treated with enalapril $\left(10 \mathrm{mg} \mathrm{kg}^{-1} \mathrm{day}^{-1}\right.$, mixed in the diet; SHRE or WKYE) for one month. After treatment, the volume-sensitive reflex was evaluated in each group by determining the decrease in magnitude of the efferent renal sympathetic nerve activity (RSNA) produced by acute isotonic saline volume expansion. Chemoreflex sensitivity was evaluated by examining the bradycardia response elicited by phenyldiguanide administration. Cardiac hypertrophy was determined from the left ventricular/body weight (LV/ $\mathrm{BW}$ ) ratio. Volume expansion produced an attenuated renal sympathoinhibitory response in SHR as compared to WKY rats. As compared to the levels observed in normotensive WKY rats, however, enalapril treatment restored the volume expansion-induced decrease in RSNA in SHRE. SHR with established hypertension had a higher $\mathrm{LV} / \mathrm{BW}$ ratio (45\%) as compared to normotensive WKY rats. With enalapril treatment, the LV/BW ratio was reduced to $19 \%$ in SHRE. Finally, the reflex-induced bradycardia response produced by phenyldiguanide was significantly attenuated in SHR compared to WKY rats. Unlike the effects on the volume reflex, the sensitivity of the cardiac chemosensitive reflex to phenyldiguanide was not restored by enalapril treatment in SHRE. Taken together, these results indicate that the impairment of the volume-sensitive, but not the chemosensitive, reflex can be restored by treatment of SHR with enalapril. It is possible that by augmenting the gain of the volume-sensitive reflex control of RSNA, enalapril contributed to the reversal of cardiac hypertrophy and normalization of arterial blood pressure in SHR.
Key words

- Volume-sensitive reflex

- Cardiac hypertrophy

- Chemosensitive reflex

- SHR

- Enalapril 
The reflex control of the circulation depends not only on arterial baroreceptors, but also on receptors located in the cardiopulmonary region. In this regard, the cardiopulmonary reflex specifically controls the activity of sympathetic nerve fibers. In rats, prolonged stimulation of the volume-sensitive cardiopulmonary reflex produced by isotonic saline volume expansion inhibits efferent renal sympathetic nerve activity (RSNA) $(1,2)$.

The sympathetic nervous system is known to participate in the regulation of arterial blood pressure by its influence on total peripheral resistance. In addition, through alterations in the renal handling of sodium and water, changes in central sympathetic outflow to the kidneys help maintain homeostasis of blood volume and consequently arterial blood pressure (3). Thus, any condition that leads to an increase in the basal level of efferent RSNA could lead to an impairment of volume homeostasis due to avid sodium and water retention by the kidneys. Over time these effects can lead to fluid and electrolyte derangement and contribute to the development of hypertension (4).

Cardiopulmonary receptors are stimulated by changes in cardiac filling pressure and by chemical agents (5). With respect to the latter, the activation of the chemosensitive reflex is referred to as the Bezold-Jarisch reflex (6). Abnormalities are known to exist in both chemo- (7) and volume-sensitive (8) reflexes in spontaneously hypertensive rats (SHR). In addition, in this animal model of hypertension (9) and in hypertensive humans (10), the gain of the cardiopulmonary reflex is altered by the cardiac hypertrophy that also develops. Therefore, the aim of the present study was to determine whether chronic antihypertensive treatment with enalapril would restore the sensitivity of the cardiopulmonary reflexes in SHR by normalizing arterial blood pressure and reducing cardiac hypertrophy.

Four-month-old male SHR and corre- sponding normotensive Wistar-Kyoto (WKY) rats were obtained from our institution. The rats were housed in individual cages, received water ad libitum, and were maintained in a room with a constant temperature and a 12-h light/dark cycle. Untreated SHR $(\mathrm{N}=8)$ and WKY rats $(\mathrm{N}=8)$ were fed a normal diet. Treated SHR (SHRE, $\mathrm{N}=8$ ) and WKY rats (WKYE, $\mathrm{N}=6$ ) received enalapril (10 $\mathrm{mg} \mathrm{kg}^{-1}$ day $^{-1}$; Sigma Chemical Co., St. Louis, MO, USA) mixed in the diet for one month. At the end of the treatment period, the rats were anesthetized with tribromoethanol $(2.5 \mathrm{mg} / \mathrm{kg}$, Aldrich Chemical Co., Milwaukee, MN, USA) and a PE 50 catheter was placed into the femoral artery and vein. Eleven hours later, mean arterial pressure (MAP) and heart rate (HR) were measured in conscious animals using a pressure transducer (Grass Instrument Div., Warwick, NY, USA; model PT 300) coupled to a Biopac System (MP100, Santa Barbara, CA, USA).

The first protocol was used to examine the chemosensitive limb of the cardiopulmonary reflex. The sensitivity of the chemoreflex was studied by examining the maximum bradycardia response that was elicited by the $i v$ bolus injection of increasing doses of phenyldiguanide (PDG, 0.9 to $2.1 \mathrm{nmol} / \mathrm{kg}$; Sigma). In the second protocol, the rats were anesthetized with urethane $(1 \mathrm{~g} / \mathrm{kg}$, ip $)$ and the left kidney was exposed via a retroperitoneal approach through a left flank incision. Using a dissecting microscope (M 900 DF, Vasconcellos, São Paulo, SP, Brazil) a renal nerve was identified, isolated, and carefully dissected. The renal nerve branch was then placed on a bipolar platinum wire electrode and gelled with silicone. Extracellular action potentials were recorded with an AC amplifier (NL 104, NeuroLog, Digitimer, Welwyn Garden City, Hertfordshire, England) connected to a high impedance headstage (NL 100). The amplified signals were filtered (NL 126), connected to an audio amplifier (NL 120) and displayed on an oscilloscope 
(Tektronix 2205, São Paulo, SP, Brazil). The data were processed using a spike trigger (NL 200) and a ratemeter (NL 256) and displayed on a Biopac System (MP100). All data were digitized and stored (Digital MTE 46602 Tape Stream) for further analysis (Acknowledge for Windows; Biopac Inc.). After completion of surgical preparation and equilibration, the volume-sensitive reflex was stimulated with an infusion of a $0.9 \%$ saline load that corresponded to $1.5 \%$ of the animal's body weight (i.e., approximately 2$2.5 \mathrm{ml} / \mathrm{min}$ ). The volume-sensitive limb of the cardiopulmonary reflex was quantified by measuring the maximum decrease in integrated RSNA (IRSNA). In these studies, the acute volume expansion used was sufficient to evoke a plateau inhibition of RSNA. MAP was simultaneously monitored during the infusion. After each experiment, the extent of cardiac hypertrophy was determined for each animal by calculating the ratio of left ventricular wet weight (including the septum) and body weight (LV/BW).

Data for basal MAP, HR, LV/BW (index of cardiac hypertrophy) and changes in these parameters evoked by acute volume expansion (the volume-sensitive reflex) were subjected to one-way analysis of variance (ANOVA). Data for changes in HR produced by phenyldiguanide (the chemosensitive reflex) were analyzed statistically by two-way ANOVA. The post-hoc test used for each case was the Tukey test for multiple comparisons. All experiments were carried out in accordance with the guiding principles for biomedical research involving animals as stated by the Federation of Brazilian Societies of Experimental Biology.

The body weight of the animals tested was lower in SHR $(290 \pm 13 \mathrm{~g}, \mathrm{P}<0.05)$ than in $\mathrm{WKY}$ rats $(384 \pm 13 \mathrm{~g})$. Compared to each of these respective control groups, the weights of animals treated with enalapril were also significantly different $(\mathrm{SHRE}=242 \pm 8 \mathrm{~g}$, $\mathrm{P}<0.01 ; \mathrm{WKE}=342 \pm 8 \mathrm{~g}, \mathrm{P}<0.01)$. As shown in Figure 1 (top panel), the mean
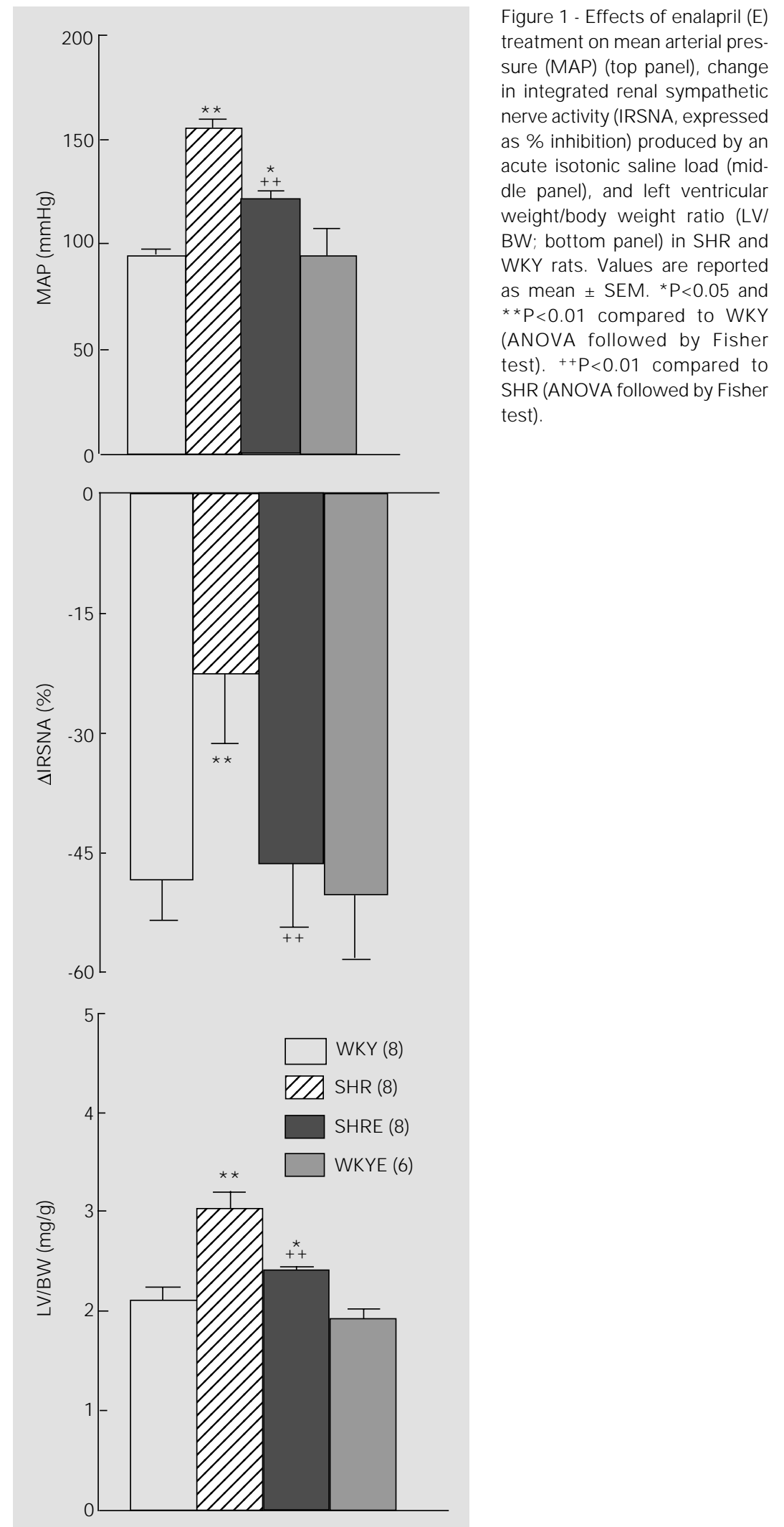
arterial pressure of untreated SHR was significantly higher $(155 \pm 6 \mathrm{mmHg}, \mathrm{P}<0.01)$ than that of untreated WKY rats $(97 \pm 3$ $\mathrm{mmHg}$ ). Long-term antihypertensive treatment with enalapril reduced the MAP in SHRE $(123 \pm 5 \mathrm{mmHg}, \mathrm{P}<0.01)$, but not in WKYE (93 $\pm 5 \mathrm{mmHg}$ )(Figure 1, top panel).

The middle panel of Figure 1 depicts the effects of an isotonic saline load (stimulation of the volume-sensitive reflex) on the change in RSNA (mean values are expressed as \% inhibition; IRSNA). SHR showed an attenuated IRSNA response compared to

\begin{tabular}{|c|c|c|c|c|c|}
\hline \multirow[t]{2}{*}{ Groups } & \multicolumn{5}{|c|}{ Phenyldiguanide $(\mathrm{nmol} / \mathrm{kg})$} \\
\hline & 0.9 & 1.2 & 1.5 & 1.8 & 2.1 \\
\hline WKY $(\mathrm{N}=8)$ & $-33 \pm 9$ & $-44 \pm 7$ & $-54 \pm 7$ & $-63 \pm 6$ & $-69 \pm 4$ \\
\hline WKYE $(N=6)$ & $-36 \pm 12$ & $-64 \pm 13$ & $-71 \pm 14$ & $-73 \pm 6$ & $-67 \pm 7$ \\
\hline $\mathrm{SHR}(\mathrm{N}=8)$ & $-20 \pm 7$ & $-26 \pm 7^{*}$ & $-42 \pm 8^{*}$ & $-46 \pm 5^{*}$ & $-40 \pm 6^{*}$ \\
\hline SHRE $(N=8)$ & $-20 \pm 6$ & $-27 \pm 5^{*}$ & $-34 \pm 6^{*}$ & $-47 \pm 6^{*}$ & $-46 \pm 6^{*}$ \\
\hline
\end{tabular}

Figure 2 - Responses of heart rate to intravenous injections of phenyldiguanide in enalapriltreated SHR (filled squares) and WKY (open circles), compared with untreated SHR (open squares) and WKY (filled circles). Values are reported as mean \pm SEM and symbols indicate differences in the reflex bradycardic response compared to WKY and WKYE (*P<0.05; ANOVA followed by Fisher test).

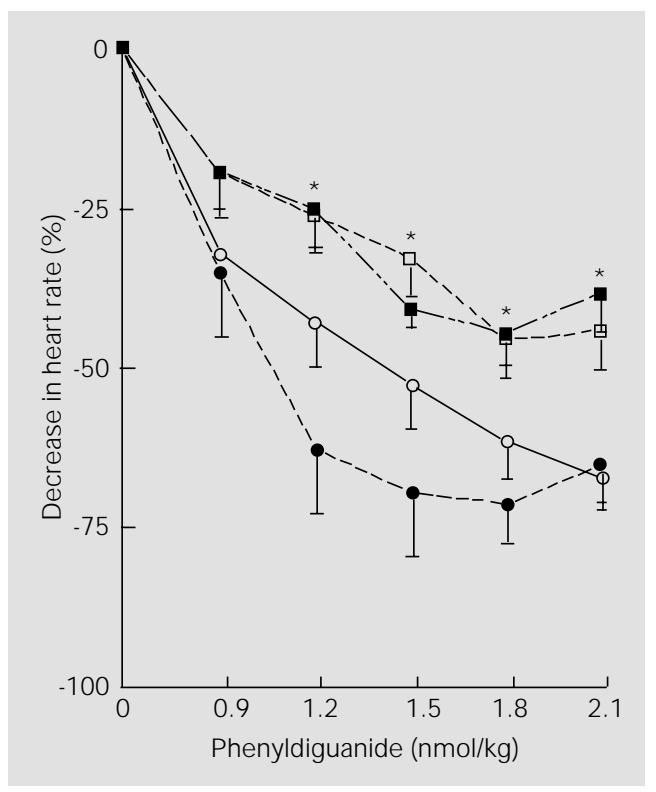

WKY rats. In contrast, long-term treatment with enalapril restored the ability of cardiopulmonary receptors to modulate RSNA. This is supported by the observation that volume expansion elicited in SHRE an IRSNA response similar to that observed in normotensive WKY. Enalapril treatment did not alter the IRSNA response to volume expansion in WKYE. In these studies, MAP and HR were not significantly altered by volume expansion in any group (data not shown).

As shown in Figure 1 (bottom panel), the LV/BW ratio of SHR was approximately $45 \%$ higher than that of age-matched normotensive $\mathrm{WKY}$ rats $(\mathrm{LV} / \mathrm{BW}=2.08 \mathrm{mg} / \mathrm{g})$. The LV/BW ratio was significantly lower in SHRE than in untreated SHR. In SHRE, the $\mathrm{LV} / \mathrm{BW}$ ratio was only $19 \%$ higher than that of enalapril-treated WKYE. The latter finding indicates that the extent of ventricular hypertrophy was substantially reduced by enalapril treatment although antihypertensive treatment with enalapril did not alter the LV/BW ratio in WKYE $(1.98 \mathrm{mg} / \mathrm{g}$; Figure 1, bottom panel).

In WKY rats, the $i v$ injection of PDG produced a dose-dependent reduction in HR (Table 1 and Figure 2). Compared with WKY and WKYE rats, the PDG-induced bradycardia was significantly attenuated $(\mathrm{P}<0.05)$ in the SHR groups, beginning at the second dose tested. No significant differences were observed between WKY and WKYE groups. Unlike the effects of antihypertensive treatment on the volume-sensitive reflex, the cardiac chemosensitive reflex was not altered by the long-term administration of enalapril to SHR.

The major finding of this study is that the impaired gain of the volume-sensitive reflex control of RSNA in SHR is restored by the chronic administration of enalapril. Although not specifically studied, augmentation of the gain of this branch of the cardiopulmonary reflex may contribute to the reduction in arterial pressure observed in SHR treated with enalapril. In addition, the present find- 
ings demonstrate the relationship between the gain of the volume-sensitive reflex control of RSNA and left ventricular hypertrophy.

In the present study the chemosensitive reflex was quantified by measuring the maximum reduction in HR evoked by $i v$ bolus injection of phenyldiguanide. Quantification was performed in this manner since there are two components of the bradycardia that occurs from activation of both the baroreceptor and the Bezold-Jarisch reflex. In this regard, activation of either reflex evokes an increase in vagal drive and a reduction in sympathetic outflow to the heart $(11,12)$. There are certain differences, however, in the extent to which each branch of the cardiopulmonary reflex affects the vagal component or sympathetic withdrawal. For instance, during acute volume expansion the volume-sensitive receptors are stimulated and lead predominantly to an inhibition of central sympathetic outflow (i.e., inhibition of RSNA). On the other hand, during conditions in which the Bezold-Jarisch reflex is activated (i.e., phenyldiguanide administration), activation of cardiac vagal motoneurones (i.e., the parasympathetic component) tends to predominate and mediates a transient bradycardia (11). In the latter case, a role for the activation of parasympathetic pathways is suggested since the chemoreceptor-mediated reduction in HR is greatly attenuated by atropine pretreatment (13). In our study, enalapril treatment normalized the volumesensitive but not the chemosensitive branch of the cardiopulmonary reflex in SHR. Therefore, cardiopulmonary reflexes with different sensory properties may be affected in separate ways in these animals. Taken together, these results support the view that the chemosensitive and volume-sensitive afferent pathways are likely to be separate entities (14).

Although we cannot exclude the possibility that the impaired volume-sensitive reflex in SHR depends on alterations of other portions of the reflex arc, an explanation for this impairment could be the cardiac hypertrophy observed in these genetically hypertensive animals. Studies indicate that there is marked elevation of left atrial pressure in hypertensive rats (15). Cellular hypertrophy is a general phenomenon in hypertensive animals, both in the left ventricle and in the resistance vessels. It is therefore likely that this elevated left atrial pressure will induce hypertrophy of the atrial wall, with a consequent reduction of wall distensibility. This distensibility of the left atrium will then probably be the main reason for the resetting of the cardiopulmonary receptors (9). Based on this premise, it would be anticipated that a reduction in cardiac hypertrophy in SHR may be associated with a concomitant normalization of the sensitivity of the reflex control of RSNA. In fact, this association was demonstrated in the present study. Whereas enalapril reduced the $\mathrm{LV} / \mathrm{BW}$ ratio (an index of cardiac hypertrophy) and improved the volume-sensitive reflex in SHRE, it did not alter arterial pressure, the LV/BW ratio or cardiopulmonary reflex function in normotensive rats. Furthermore, Grassi and coworkers (10) reported that in hypertensive subjects with left ventricular hypertrophy, antihypertensive treatment resulted in the regression of the hypertrophy and an associated improvement in the cardiopulmonary reflex response to modulate vascular and humoral stimuli. On the other hand, additional findings suggest that the impairment of the chemosensitive reflex in hypertensive animals or humans could be due to other factors independent of cardiac hypertrophy or high blood pressure. For instance, studies by Thomas et al. (14) have shown that the chronic administration of perindopril (an angiotensin-converting enzyme inhibitor) to young SHR (i.e., prior to the development of hypertension), prevented hypertension and the development of left ventricular hypertrophy. Based on our data and theirs, despite regression of hypertension and cardiac hy- 
pertrophy, antihypertensive treatment did not normalize the function of the Bezold-Jarisch reflex. Further studies are required to clarify this impairment of the Bezold-Jarisch reflex.

In conclusion, the present study demonstrated that chronic administration of enalapril reduced arterial blood pressure and cardiac hypertrophy in SHR. In addition, enalapril treatment improved volume gain but not the chemosensitive cardiopulmonary reflex. Augmentation of the volume-sensitive control of RSNA in hypertensive animals may contribute to the restoration of normal arterial pressure homeostasis.

\section{References}

1. Veelken R, Sawin LL \& DiBona GE (1989). Dissociation of renal and excretory responses to volume expansion in prehypertensive Dahl salt-sensitive and salt-resistant rats. Hypertension, 13: 822-827.

2. Veelken $R$, Hilgers KF, Leonard $M$, Ruhe J , Serogin K, Mann J FE \& Luft FC (1993). A highly selective cardiorenal, serotoninergic 5- $\mathrm{HT}_{3}$-mediated reflex in rats. American J ournal of Physiology, 264: H1871$\mathrm{H} 1877$.

3. DiBona GF (1989). Neural control of renal function: cardiovascular implications. Hypertension, 13: 539-548.

4. Katholi RE (1983). Renal nerves in the pathogenesis of hypertension in experimental animals and humans. American J ournal of Physiology, 245: F1-F14.

5. Zucker IH (1989). Left ventricular receptors: physiological controllers or pathological curiosities. Basic Research in Cardiology, 81: 539-557.

6. Mark AL (1983). The Bezold-J arisch reflexes revisited: clinical implications of in- hibitory reflexes originating in the heart. J ournal of the American College of Cardiology, 1: 90-102.

7. Thorton RM, Wyss J M \& Oparil S (1989). Impaired reflex responses to volume expansion in $\mathrm{NaCl}$-sensitive spontaneously hypertensive rats. Hypertension, 14: 518523.

8. Widdop RE, Verbene AJ M, J arrott B \& Louis WJ (1990). Impaired arterial baroreceptor reflex and cardiopulmonary vagal reflex in conscious spontaneously hypertensive rats. J ournal of Hypertension, 8: 269-275.

9. Thorén $\mathrm{P}$, Noresson $\mathrm{E} \&$ Ricksten SE (1979). Resetting of cardiac C-fiber endings in the spontaneously hypertensive rat. Acta Physiologica Scandinavica, 107: 13-18.

10. Grassi G, Giannattasio C, Cléroux J, Cuspidi C, Sampieri L, Bolla GB \& Mancia G (1988). Cardiopulmonary reflex before and after regression of left ventricular hypertrophy in essential hypertension. Hy- pertension, 12: 227-237.

11. Spyer KM (1982). Central nervous integration of cardiovascular control. J oumal of Experimental Biology, 100: 109-128.

12. Hatab AS, Futuro-Neto HA \& Pires J GP (1996). Are 5-HT 1 A receptors involved in the control of reflex bradycardia induced by intracoronary injection of contrast medium in humans? Brazilian J ournal of Medical and Biological Research, 29: 769772.

13. Chianca J r DA \& Machad BH (1997). Microinjection of NMDA antagonist into the NTS of conscious rats blocks the BezoldJ arisch reflex. Brain Research, 718: 185188.

14. Thomas J C, Head GA \& Woods RL (1998). ANP and bradycardic reflexes in hypertensive rats. Influence of cardiac hypertrophy. Hypertension, 32: 548-555.

15. Noresson E, Ricksten S \& Thorén P (1979). Left atrial pressure in normotensive and spontaneously hypertensive rats. Acta Physiologica Scandinavica, 107: 9-12. 